\section{Acrodermatitis Enteropathica as a Presentation of Cystic Fibrosis in an Infant}

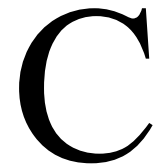
ystic Fibrosis (CF) in an autosomal recessive disease due to mutation of CFTR gene on chromosome 7 which is involved in ionic transport across membranes. Children with CF commonly present with recurrent or persistent respiratory infections and exocrine pancreatic insufficiency, however, the clinical presentation can be varied.

A seven-month-old girl was referred with non-healing skin lesions and failure to thrive. She was born to third degree consanguineous parents with uneventful perinatal period. She was asymptomatic with normal weight gain till 4 months of age when she developed perianal maculopapular rashes which failed to improve with topical steroids. Her weight started to plateau. There were no respiratory infections. Acrodermatitis enteropathica was suspected at 7 months of age. Serum zinc levels were low (46 $\mu \mathrm{g} / \mathrm{dL})$. Her length and weight were below the 3rd centile for age as per WHO growth charts. She was irritable and had sparse hair. There were no dysmorphic features or congenital anomalies. She had extensive maculo-papular erythematous rashes, especially in the perianal region with pitting pedal edema. During examination, the baby passed stools which were oily. A detailed family history revealed two siblings deaths in early infancy with recurrent respiratory symptoms. Investigations indicated hemoglobin $8.3 \mathrm{~g} / \mathrm{dL}$, aspartate aminotransferase $156 \mathrm{U} / \mathrm{L}$, alanine aminotransferase 52 $\mathrm{U} / \mathrm{L}$, and serum albumin $2.1 \mathrm{~g} / \mathrm{dL}$. She was started on oral zinc supplementation and protein rich diet. CF was suspected as the child had oily stools, transaminitis and sibling deaths with recurrent respiratory symptoms. She was started on fat soluble vitamin supplementation with pancreatic lipase. Genetic analysis showed homozygous mutation on exon 14 of the CFTR gene (p.Arg709Ter), which was deemed pathogenic for cystic fibrosis. After three months, her rashes had disappeared and weight was between the $3 \mathrm{rd}-10$ th centiles.

Acrodermatits enteropathica is a rare autosomal recessive disorder of zinc absorption. Affected infants usually present with erythematous maculo-papular rash typically in the perianal region, diarrhoea and alopecia. The presentation usually coincides with initiation of complementary feeding. Other symptoms include irritability, conjunctivitis and nail changes. Acrodermatitis enteropathica has been described as a presenting feature of $\mathrm{CF}$ secondary to zinc malabsorption with pancreatic insufficiency. Hypoalbuminemia is likely to further exacerbate zinc deficiency. Most children with $\mathrm{CF}$ present with recurrent respiratory infections and steatorrhea. The index infant presented with FTT, oily stools as well as a family history suggestive of CF. The diagnosis of CF is more frequently considered in Indian children with increasing access to genetic testing. Delta 508, which is the commonest mutation associated with $\mathrm{CF}$ in the Caucasian population, is seldom found in Indian children. The index patient had a homozygous nonsense variant at exome 14 which results in a stop codon and premature truncation of protein at codon 709 .

In conclusion, $\mathrm{CF}$ is associated with a wide range of presenting features which includes acrodermatitis enteropathica. A high index of suspicion, detailed history (including family history) and a thorough clinical examination will aid in directing investigations towards confirmation of the diagnosis. Genetic testing is often required for confirming diagnosis in infants with unusual presentation

Contributors: MM: contributed to data collection, writing and editing; RR: contributed to design, content and review of content; MS: contributed to data collection, writing and editing. All authors approved the final version of manuscript, and are accountable for all aspects related to the study.

Funding: None; Competing interest: None stated.

Manoj Madhusudan, Radhika Raman* AND Malathi Sathyasekaran, Kanchi Kamakoti CHILDS Trust Hospital, Chennai, India. *doc.rads15@gmail.com

\section{REFERENCE}

1. Zedek D, Morrel DS, Graham M, Goodman D, Groben P. Acrodermatits enteropathica like eruptionsand failure to thrive as presenting signs of cystic fibrosis. J Am Acad Dermatol. 2008;58;S5-8.

2. Mei-Zahav M, Solomon M, Kawamura A, Coates A, Durie P. Cystic Fibrosis presenting as kwashiokor in a Sri Lankan infant. Arch Dis Child. 2003;88:724-5.

3. Estivill X, Bancells C, Ramos C, Biomed CF. Mutation analysis consortium geographic distribution and regional origin of 272 cystic fibrosis mutations in European Populations. Hum Mutat. 1997;10:135-54. 